\title{
Transmisson Hub and Terminals for Point to Multipoint W-band TWEETHER System
} \author{
Viktor Krozer ${ }^{7}$, Rosa Letizia ${ }^{1}$, Marc Marilier ${ }^{8}$, Antonio Ramirez ${ }^{8}$, Ralph Zimmerman ${ }^{5}$ \\ ${ }^{1}$ Lancaster University, Lancaster LA1 4YW, UK \\ email: c.paoloni@lancaster.ac.uk \\ ${ }^{2}$ When-AB, Paris, France \\ ${ }^{3}$ Thales Electron Devices, Vélizy 78140, France \\ ${ }^{4}$ Bowen, Paris, France \\ ${ }^{5} \mathrm{HF}$ Systems Engineering GmbH, Kassel 34123, Germany \\ ${ }^{6}$ LTCI, Télécom ParisTech, Université Paris-Saclay, France \\ ${ }^{7}$ Goethe University of Frankfurt, Frankfurt 60323, Germany, \\ ${ }^{8}$ OMMIC S.A.S., Limeil Brevannes 94453, France \\ ${ }^{9}$ Fibernova Systems, Valencia 46022, Spain
}

Claudio Paoloni ${ }^{1}$, François Magne ${ }^{2}$, Frédéric André ${ }^{3}$, Joël Willebois ${ }^{4}$, Quang Trung Le ${ }^{5}$, Xavier Begaud ${ }^{6}$, Giacomo Ulisse ${ }^{7}$,

\begin{abstract}
The European Commission Horizon 2020 TWEETHER project will conclude the activity on September 2018 with the release of one transmission hub and three network terminal equipment for enabling the first W-band, 92 - $95 \mathrm{GHz}$, point to multipoint system, for high capacity backhaul and fixed access. The ambition of the project is to develop the European technology for a breakthrough in millimeter wave wireless networks, by introducing the use of traveling wave tubes to achieve the required transmission power for covering, by low-gain antennas, wide area sectors with radius longer than $1 \mathrm{~km}$. The lack of transmission power has so far prevented the use of point to multipoint distribution at millimeter waves. At $\mathrm{W}$-band, the $3 \mathrm{GHz}$ bandwidth provides more than 10 Gbps capacity, and $4 \mathrm{Gbps} / \mathrm{km}^{2}$ area capacity for small cells backhaul, with flexible allocation of the base stations and low total cost of operation. The TWEETHER system is also designed to provide high throughput fixed access. This paper will describe the latest results and the technological advancements the project generated, bringing Europe at the state of the art for point to multipoint millimeter wave wireless networks.
\end{abstract}

Keywords-Point-to-multipoint, TWT, MMIC, radio, W-band, millimeter waves, backhaul, access, terminal transmission hub;

\section{INTRODUCTION}

The use of millimeter waves to distribute high capacity wireless is considered the most promising solution to solve the huge increase of wireless traffic [ $1-4]$. Presently, a number of manufacturers produce E-band point-to-point (PtP) front ends with multigigabit per second data rate at an affordable price. However, PtP systems need two front ends with high gain antennas for each link. If $\mathrm{N}$ links are assumed, the total number of front end is $2 \mathrm{~N}$. The PtP systems are very effective for connecting two sites, but are not the most cost effective solution when a high number of terminals must to be connected, e.g. dense small cell deployment. Point to multipoint (PmP) distribution is demonstrated of flexible installation and lower Total Cost of Operation (TCO) [5, 6]. N links are served by one transmission hub and $\mathrm{N}$ terminals, with practically half of the front-ends needed at E-band. The high atmosphere attenuation at millimeter waves and the requirement of availability above $99.95 \%$ in the rain zone of operation (typically $\mathrm{K}$ ) are demanding design parameters. The link budget requires a high combined value of antenna gain plus transmission power. Whilst antennas can be designed up to $50 \mathrm{dBi}$ gain, solid state amplifiers are intrinsically limited to about than 1 Watt continuous wave above the $\mathrm{V}$ - band (about $70 \mathrm{GHz}$ ) [7]. High directive antennas could have diameters of a several tens of centimeters. The Point to multipoint needs a low gain antenna. In case of backhaul of high density cell distribution, the use of frequencies higher than the E-band has the advantages of the smaller footprint related to the shorter wavelength. In particular, if we consider the W-band $(92-110 \mathrm{GHz})$ the wavelength is $15 \%$ shorter than E-band. The W-band was already investigated for satellite communications due to the relatively low attenuation [8] in comparison to other millimeter wave bands. However, the requirements for a Wband front end are very demanding due to the atmosphere attenuation much higher than at microwaves, the lack of adequate transmission power and the difficulty of an easy and affordable assembly of the components.

The European Commission H2020 TWEETHER project, "Traveling wave tube based W-band wireless networks with

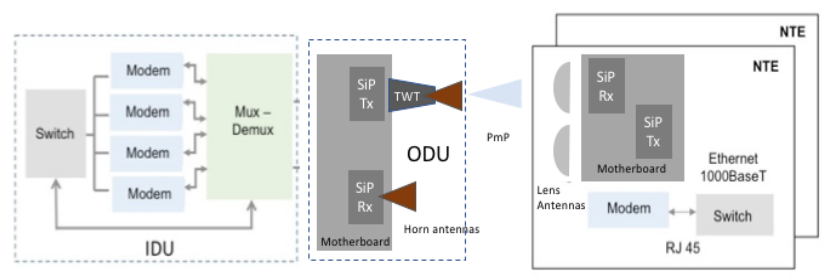

Fig. 1 Schematic of the W-band Point to multipoint TWEETHER system. 


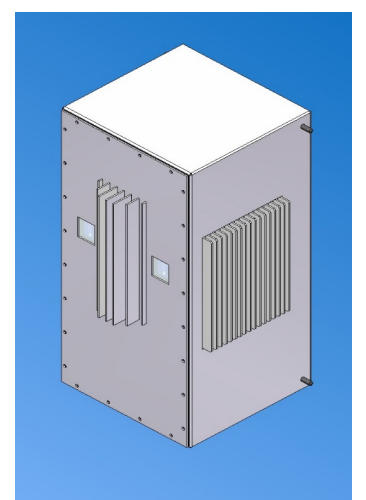

a)

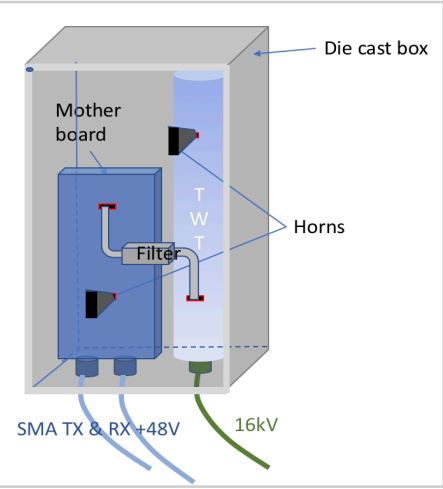

b)

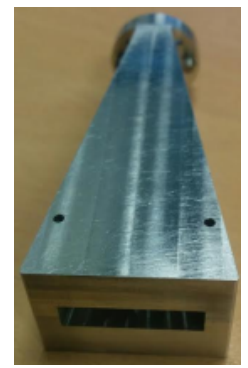

c)

Fig.2 a) External view of the Transmission Hub Out Door Unit; b) Internal structure of the Transmission Hub Out Door Unit; c) Low gain horn antenna

high data rate distribution, spectrum \& energy efficiency", aims to offer novel solutions to enable PmP at W-band, in the 92-95 $\mathrm{GHz}$ band. The main novelty is the use of a high-power Traveling Wave Tube (TWT) with more than $40 \mathrm{~W}$ saturated power, to overcome the atmosphere attenuation and to achieve a propagation at ground level longer than one kilometer on a wide area sector $[9,10]$. The TWEETHER performance would not be possible by using solid state power amplifiers. The point to multipoint at $\mathrm{W}$-band is a breakthrough in the field of wireless communications. The use of low gain antenna typical of PmP can be supported only by the high transmission power TWTs can provide.

A few TWTs at W-band are still at prototype stage in laboratories in US and China [11]. The effort of the TWEETHER Consortium has been to develop a technology for large production of TWTs, compatible with the available microfabrication technologies, in particular high accuracy $\mathrm{CNC}$ milling.

In the following, the Transmission Hub and the Network Terminal Equipment for the first Point to multipoint W-band wireless system will be described, with emphasis on the technology challenges.

\section{TWEETHER POINT TO MULTIPOINT SYSTEM}

The TWEETHER point to multipoint system at W-band consists of a Transmission Hub (TH) and a number of Network Terminal Equipment (NTE) distributed in a sector area for

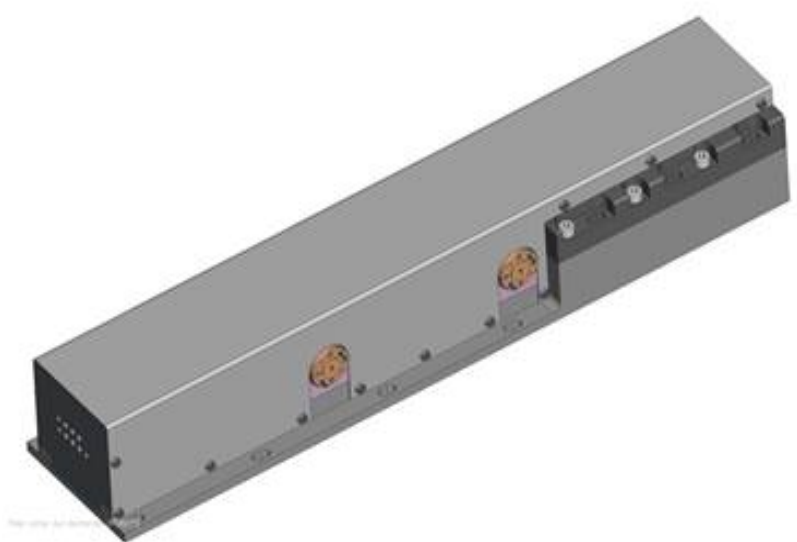

Fig.3 TWT enclosure

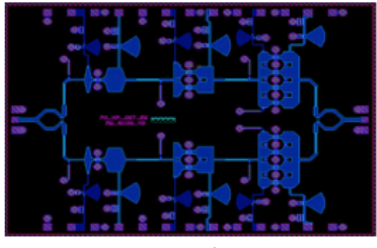

a)

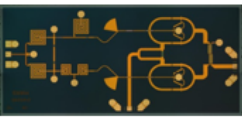

c)

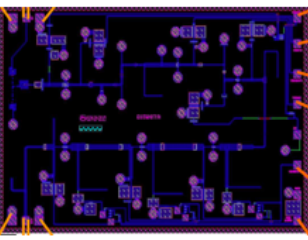

b)

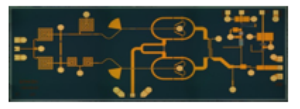

d)

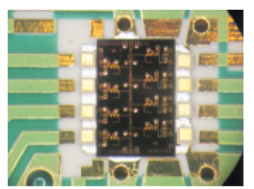

e)

Fig. 4 W-band chipset: a) power amplifier, b) 8x multiplier, c) downconverter, d) upconverter, e) low noise amplifier. T

TABLE I. MMIC CHIPSET SPECIFICATIONS

\begin{tabular}{|c|c|c|c|c|c|c|c|c|}
\hline MMIC & $\mathrm{G}$ & $\mathrm{P} 1$ & $\mathrm{OIP} 3$ & $\mathrm{~S} 22$ & $\mathrm{~S} 11$ & $\mathrm{NF}$ & Spurious & Image \\
\hline Unit & $\mathrm{dB}$ & $\mathrm{dBm}$ & $\mathrm{dBm}$ & $\mathrm{dB}$ & $\mathrm{dB}$ & $\mathrm{dB}$ & $\mathrm{dBc}$ & $\mathrm{dBc}$ \\
\hline X 8 & 2 & 11 & & 10 & 8 & 4 & -30 & \\
\hline UC & 2 & 2 & 10 & 10 & 9 & 4 & $-6 \mathrm{LO}$ & -12 \\
\hline PA & 22 & 22 & 30 & 10 & 10 & 6 & \multicolumn{2}{|c|}{ PAE $10 \%$} \\
\hline LNA & 22 & 0 & & 10 & 10 & 3 & \multicolumn{2}{|c|}{} \\
\hline DC & 10 & 0 & & 13 & 15 & 4 & -12 LO & -25 \\
\hline
\end{tabular}

backhaul or fixed access (Fig. 1). A possible scenario is to assign the $3 \mathrm{GHz}$ available at $\mathrm{W}$-band evenly among three operators, 
each operator having $1 \mathrm{GHz}$ bandwidth. The operator would allocate $500 \mathrm{MHz}$ per sector, deploying with a frequency reuse of 2. Each sector will distribute a multiplex of up to ten $40-\mathrm{MHz}$ channels or five $80-\mathrm{MHz}$ channels, or a combination of them. A minimum of one NTE per channel on each sector will be deployed. At each NTE, one of the channels of the multiplex will be demodulated.

\section{TRANSMISSION HUB}

The Transmission Hub (TH) comprises an Indoor Unit (IDU) and an Outdoor Unit (ODU). The IDU hosts a COTS (Commercial-Off-The-Shelf) GbE switch, a stack of SISO (Single In Single Out) 802.1 lac modems with separated Tx and $\mathrm{Rx}$ connectors and a C-band multiplexer/demultiplexer unit. The TH ODU hosts the motherboard, the TWT and two low gain horn antennas (one for the receiver and one for the transmitter) designed to cover a specific sector angle. The transmitted low-gain horn antenna generated a wide beam to connect the NTEs placed where needed in the covered areas.

\section{A. Structure}

The transmission hub ODU (Fig. 2) consists of a metal case (Fig. 2a) including a motherboard with the transmitter and receiver, both built as Systems in Package (SIP) (Fig. 2b). The transmitter SIP is connected to the Traveling Wave Tube (TWT) amplifier. The output port of the TWT is connected to the low

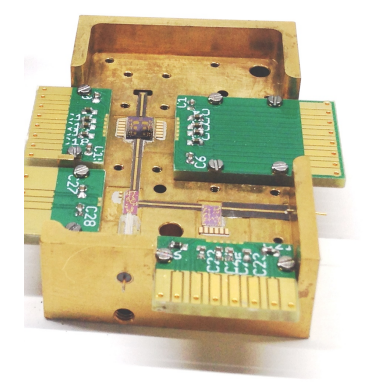

a)

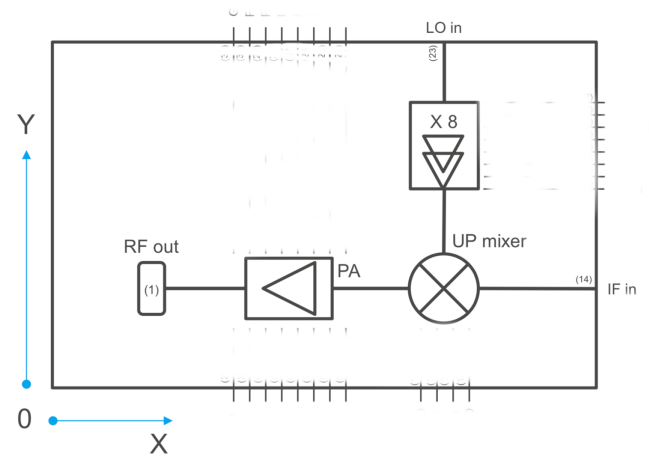

b)

Fig. 5 Transmitter System in Package a) photos of realised SIP, b) internal layout gain horn antenna (Fig. 2c). The input of the receiver SIP is connected to a similar horn antenna. The antennas are sealed by radomes. The motherboard of the Transmission Hub Out Door Unit is connected to the IDU by low-loss coaxial cables carrying the multiplex of C-band channels generated by the stack of modems in the IDU.

The ODU motherboard is also powered through the coaxial cables, while the TWT requires a dedicated power supply housed together with the IDU and employing dedicated cables.

\section{B. Traveling wave tube}

The traveling wave tube (TWT) is the enabling device for PmP at W-band. The TWT will provide the needed transmission power to support a low gain antenna and distribute the signal in a wide angle sector with more than $1 \mathrm{~km}$ range. It is derived from the well-established technology of helix TWTs for space applications. However, the short wavelength at W-band does not allow to use helix as interaction structure with the electron beam. Novel solutions are required to fabricate the metal interaction structure, with dimensions of parts in the order of hundreds of microns and tight mechanical tolerances.

The TWT has been designed, assembled and baked. The baking phase is important to create a high vacuum level inside the tube for allowing the proper flowing of the electron beam. The next step is the measurements of the TWT performance, before to mount it in the transmission hub for outdoor deployment. The TWT is about $35 \mathrm{~cm}$ long. The electron gun has been active for more than 3000 hours to test the lifetime and the beam transmission to avoid damages and malfunctioning. The target output power will be $40 \mathrm{~W}$ saturated. The TWT will be driven with $6 \mathrm{~dB}$ back-off, providing $10 \mathrm{~W}$ output power, to provide the linearity required for 64 QAM modulation scheme [11].

\section{W-band Chipset}

A full W-band chipset has been designed and produced, by a $70 \mathrm{~nm} \mathrm{GaAs} \mathrm{process,} \mathrm{with} \mathrm{European} \mathrm{technology} \mathrm{in} \mathrm{W-band}$ (92 - $95 \mathrm{GHz})$. The specifications are listed in Table I. It includes

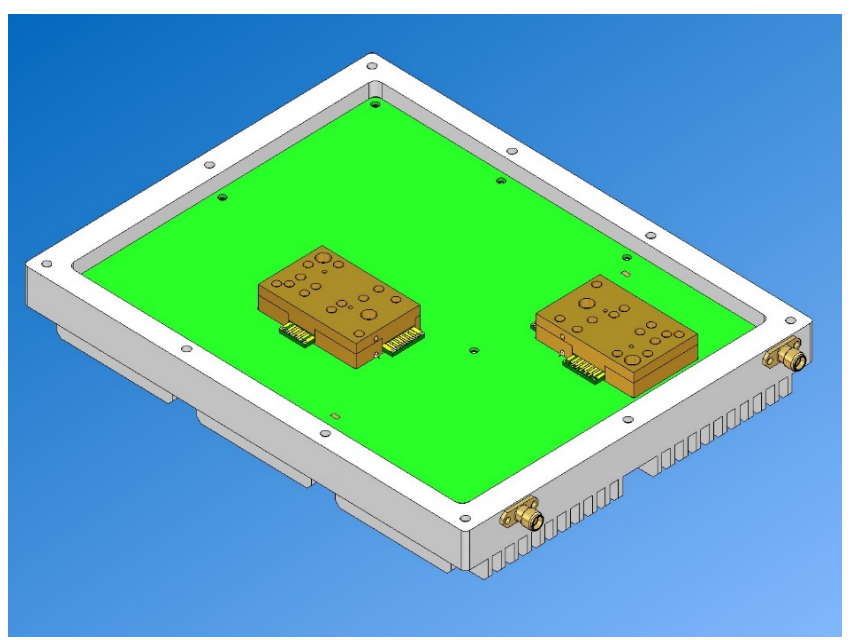

Fig. 6 Drawing of the Motherboard with the two SIPs, one for the receiver and one for the transmitter. 
a power amplifier as driver for the TWT and to provide transmission power to the Network Terminal Equipment, an up converter and a downconverter for the radio, an $8 x$ multiplier for rising in frequency the synthesizer signal and a low noise amplifier for the W-band radio receiver (Fig. 4). The chipset has been designed to be used both for the TH and the NTE, as a costeffective solution. The chipset has been tested both on wafer and in package. The most challenging specification is the output power of the power amplifier. The PA power is very important for the NTE to assure the suitable range with the available antenna for the link with the TH. About $19 \mathrm{dBm}$ were measured, lower than the $22 \mathrm{dBm}$ in the specifications. However, this value permits to achieve the required range with a lower modulation scheme. The low noise amplifier has performance at the state of the art, with $3 \mathrm{~dB}$ noise figure in a wide band around $92-95$ $\mathrm{GHz}$.

\section{System in Package}

Two Systems in Package (SIP), one for the receiver and one for the transmitter, have been fabricated (Fig. 5). Each of them includes the radio based on the $\mathrm{W}$-band chipset. The challenge was to create interconnection with low loss. The SIP substrate is at the level of the 1st layer of the motherboard to connect the IF input (Tx) or IF output (Rx) and Local Oscillator input. The MMICs are inserted in the substrate for flat bonding and soldered to the SIP support. A cap with absorber will close the SIP to avoid any parasitic radiation.

\section{E. Motherboard}

Two motherboards are in realization phase, including all the electronics of the TH and the NTE respectively (Fig. 6). The

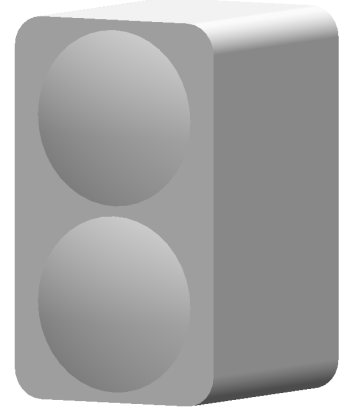

a)

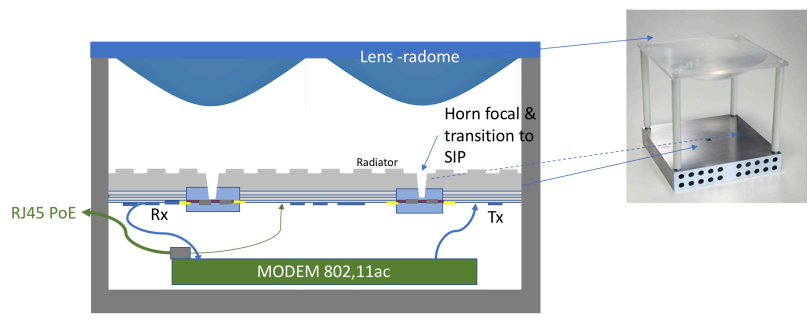

b)

Fig. 7. NTE a) case, b) internal structure with lens antennas motherboard of the TH includes two SIPs. One for the receiver connected to the horn antenna, the second for the transmitter connected to the TWT. Each motherboard has all the connections for the power supply and includes the synthesizer at $\mathrm{Ku}$ band. The synthesizer frequency is split to the SIP up converter (port LO in Fig. 5 b) and down converter, where is multiplied by 8 by a purposely designed $8 x$ multiplier in GaAs technology.

\section{NETWORK TERMINAL EQUIPMENT}

The Network Terminal Equipment (Fig. 7) hosts the W-band transmitter and receiver to establish the W-band wireless backhaul link for small cell base stations or fixed access distribution points. The NTE is very compact and of easy deployment (Fig. 7a). Its volume is less than $2 \mathrm{dm}^{3}$. It will be connected in line of sight to the TH. As the TH, this is based on a motherboard that hosts the transmission and receiver SIPs. The two SIPs are connected by two feeders to two high directivity lens antennas (Fig. 7b). The lens antennas are of easy fabrication and small diameter (about $12 \mathrm{~cm}$ ) with a gain of about $35 \mathrm{dBi}$ [12].

The chipset and the SIPs are the same used in the Transmission Hub. The difference is that the transmitter SIP in the NTE has the Power Amplifier biased for high output power.

\section{CONCLUSIONS}

The Horizon 2020 TWEETHER project has been a challenging and exciting work to develop state of the art European technology for enabling the first point to multipoint millimeter wave system above $90 \mathrm{GHz}$. For the first time a full system for outdoor point to multipoint at W-band has been designed and built. The design and fabrication of the TWT, the chipset, the SIPs and motherboards required an outstanding effort and significant novelty, to overcome the difficulties due to small dimensions and parasitic effects typical of operation frequencies close to $100 \mathrm{GHz}$. The test of the Transmission Hub and the NTE will be performed by end of September 2018 in a Field Trial involving one $\mathrm{TH}$ and three NTEs installed at different distance, the further being around $1.1 \mathrm{Km}$.

\section{ACKNOWLEDGMENT}

The project has received funding from the European Union's Horizon 2020 research and innovation program under grant agreement no 644678 .

\section{REFERENCES}

[1] Z. Pi and F. Khan, "An introduction to millimeter-wave mobile broadband systems," in IEEE Comm. Magazine, pp. 101-107, June 2011. [

[2] X. Li, et al. , "Fiber-wireless- fiber link for 100-Gb/s PDM-QPSK signal transmission at W-band," IEEE Photon. Technol. Lett., Jul. 2014.

[3] T.S. Rappaport et al., " Millimeter Wave Mobile Communications for 5G Cellular: It will work!”, IEEE Access, pp. 335-349, Mai 2013.

[4] J. Takeuchi, et. al. "10-Gbit/s Bi-directional wireless data transmission system using 120-GHz-band ortho-mode transducers," in 2012 IEEE Radio and Wireless Symposium, 2012, pp. 63-66.A. 
[5] Total cost of ownership and time breakeven of last mile data transport for MNOs and ISPs, http://www.realwireless.biz/resources/publications/.

[6] R. Taori and A. Sridharan. "Point-to-multipoint in-band mmwave backhaul for 5G networks," in IEEE Comm.s Magazine, pp. 195-201, January 2015.

[7] J. Schellenberg et al., "W-Band, Broadband 2W GaN MMIC," in IEEE MTT-S International Microwave Symposium (IMS), 2-7 June 2013

[8] Cianca E, Rossi T, Yahalom A, Pinhasi Y, Farserotu J, Sacchi C (2011). EHF for satellite communications: the new broadband frontier. PROCEEDINGS OF THE IEEE, vol. 99, p. 1858-1881, ISSN: 00189219, doi: 10.1109/JPROC.2011.2158765

[9] Paoloni, C, Magne, F, Andre, F, Begaud, X, Krozer, V, Marilier, M, Ramirez, A, Vilar, R \& Zimmerman, R, TWEETHER Future Generation
W-band backhaul and access network infrastructure and technology. in European Conference on Networks and Communications (EuCNC 2017).

[10] TWEETHER website [Online]. Available: http://www.tweether.eu

[11] Y. Hu, J. Feng, J. Cai, X. Wu, Y. Du, J. Liu, J. Chen, and X. Zhang, "Design and experimental study of a widebandwidth W- band folded waveguide continuous-wave twt," IEEE Transactions on Plasma Science, vol. 42, no. 10, pp. 3380-3386, Oct 2014,

[12] Paoloni, C, Andre, F, Kohler, S, Krozer, V, Quang, TL, Letizia, R, Sabaawi, A, Ulisse, G, Zimmerman, R. 'A traveling wave tube for 92 - 95 $\mathrm{GHz}$ band wireless applications' 41st IRMMWTHz, Denmark, Sept. 2016

[13] J.E. González, X. Begaud, B. Huyart, Q. T. Le, R. Zimmermann, F. Magne 'Millimeter Wave Antennas for Backhaul Networks', EuCAP 2017, Paris, March 2017. 